\title{
Dynamics of the N-pendulum and its application to a wave energy converter concept
}

\author{
Daniil Yurchenko - Panagiotis Alevras
}

Received: 30 July 2013 / Revised: 2 October 2013 / Accepted: 4 October 2013 / Published online: 29 October 2013

(C) Springer-Verlag Berlin Heidelberg 2013

\begin{abstract}
This paper considers a design of an N-pendulum, which represents a special case of a physical pendulum. The design of the N-pendulum not only allows uncoupling the natural frequency of the pendulum from its length, but also provides easy control of the frequency and torque. The proposed design is stimulated by the idea of developing a wave power take-off system based on the parametric pendulum. Different designs are being considered and their dynamic characteristics are investigated with respect to the feasibility of such an application. Due to the observed low frequency of ocean waves, the size of a heaving simple pendulum should span along unrealistic sizes in order to be parametrically resonant. Thus, the N-pendulum is considered and the configurations that would fulfill these frequency requirements are sought. Last, numerical simulation is conducted for an under development experimental rig aiming to test the functionality of the concept, modelling the response of the N-pendulum.
\end{abstract}

Keywords Parametric pendulum - Parametric resonance . Tri-pendulum · Power take-off · Wave energy ·

Heaving motion

\section{Introduction}

The mathematical pendulum - a lumped mass oscillating on an unstretchable massless string, is an important system in studying the theory of vibrations. This fruitful example introduces us to a sinus type nonlinearity and things related to

The first author is also known as Iourtchenko.

D. Yurchenko $\cdot$ P. Alevras $(\bowtie)$

Institute of Mechanical, Process \& Energy Engineering,

Heriot-Watt University, Edinburgh EH14 4AS, UK

e-mail:pa132@hw.ac.uk nonlinear systems, such as dependence of the pendulum's period on its amplitude, stability etc. Since it is difficult to deal with the nonlinear equation of motion, most often this equation is linearized around its low equilibrium point. Then, the squared natural frequency of the system is proportional to the acceleration of gravity and inversely proportional to the pendulum's length. Thus, the length of the pendulum should be around $10 \mathrm{~m}$ to get frequency of about $1 \mathrm{rad} / \mathrm{s}$, and it must be around $100 \mathrm{~m}$ long to lower the frequency to 0.316 $\mathrm{rad} / \mathrm{s}$. Obviously, such a bulky device is difficult to build and operate. The only well known example of such a long pendulum $(67 \mathrm{~m})$ is related to Foucault, who built it in 1851 to demonstrate the rotation of the Earth.

A physical pendulum, which natural frequency depends on the distance to its center of gravity and the moment of inertia, may be used to achieve any frequency, since its natural frequency does not depend only on the distance to the centre of gravity. In fact the natural frequency is defined by the relation between the first and the second order static moments of the pendulum. Unfortunately, in practice it is not that easy to achieve low frequency due to physical tolerance and other constrains, dictated by a specific application. For instance, if one uses a disk of radius $R$, so that its moment of inertia with respect to its center is $m R^{2} / 2$, pinned at slightly shifted from its center distance $h$, then the natural frequency of this system may be expressed as:

$\omega_{n}=\sqrt{\frac{g h}{R^{2} / 2+h^{2}}}$

The same natural frequency of $0.316 \mathrm{rad} / \mathrm{s}$ can be reached with a relatively small pendulum of $R=1 \mathrm{~m}$ and $h=5.1 \times$ $10^{-3} \mathrm{~m}=5 \mathrm{~mm}$, or $R=0.5 \mathrm{~m}$ and $h=1.27 \times 10^{-3} \mathrm{~m}=$ $1.27 \mathrm{~mm}$. It is easy to see that it is extremely difficult to 
achieve such an accuracy, since the pin's diameter $d_{p}$ may have to be significantly bigger than $h$ in order to hold the structure. Assuming that one could achieve this accuracy and tolerance in practice, it is impossible to adjust the natural frequency and torque, if needed, since they are connected and the only value, which can be adjusted is $h$. Thus, a physical pendulum with given $R$ leaves no options to develop a system with prescribed frequency and torque, which is required in some applications. The readers may confirm themselves that other cross sections, such as rectangular or square, make the design even worse in terms of the achievable low frequency.

In this paper another design is proposed, which may be considered as a special case of the physical pendulum. However, in view of the practical application, which the proposed design is intended to be used for, the $\mathrm{N}$-pendulum was proven to be much more suitable, since it allows not only making the natural frequency of the pendulum independent of its length, but also adjusting the frequency and torque of the system independently. The later is especially important in developing a wave power take-off device.

\section{$2 \mathrm{~N}$-pendulum and its natural frequency}

Let's introduce an N-pendulum - a system of $N$ rigidly connected to a common ring pendulums, which are positioned at equally spaced intervals of $2 \pi / N$, so that the bi-pendulum and tri-pendulum will have two and three arms of equal masses $m$ correspondingly. Although it is possible to create a pendulum with not-equally-spaced arms, this paper does not consider such a design. All arms except one carry a lumped mass M, positioned at distance $L_{2}$ whereas the last arm carries the same mass at distance $L_{1}$, so that $L_{1}>L_{2}$. The cases of even and odd number of arms could be considered separately.

The simplest example of an odd number of arms is the tripendulum, which is illustrated on Fig. 1. For the tri-pendulum

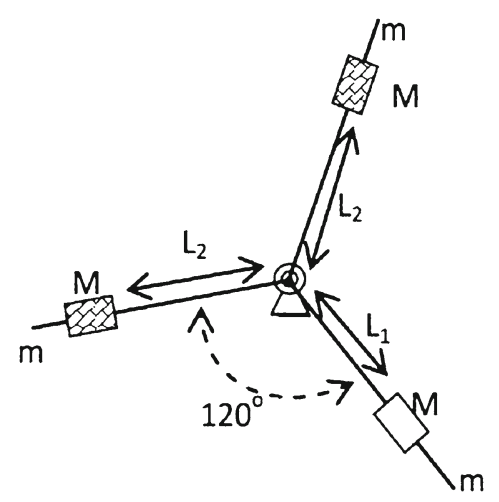

Fig. 1 Sketch of the tri-pendulum

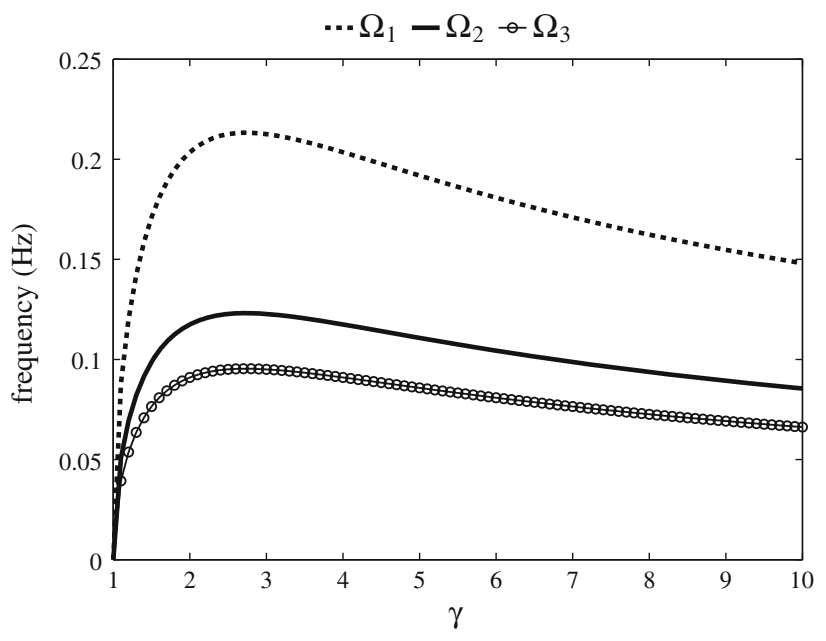

Fig. 2 Equivalent frequency of the tri-pendulum given by (3) in $\mathrm{Hz}$ : $\Omega_{1}-L_{2}=1 \mathrm{~m}, \Omega_{2}-L_{2}=3 \mathrm{~m}, \Omega_{3}-L_{2}=5 \mathrm{~m}$

the arms will be positioned at equally spaced intervals of $120^{\circ}$ so that the equation of free motion may be written as:

$$
\begin{aligned}
& {\left[3 m l^{2}+M\left(L_{1}^{2}+2 L_{2}^{2}\right)\right] \ddot{\theta}} \\
& =-M g L_{1} \sin \theta-M g L_{2} \sin \left(\frac{2 \pi}{3}+\theta\right) \\
& \quad+M g L_{2} \sin \left(\frac{2 \pi}{3}-\theta\right)
\end{aligned}
$$

It should be noted that there is no restoring force from the arms themselves, since they have equal masses and shapes, therefore they are in equilibrium. Then Eq. (2) after some simplifications will reduce to:

$\ddot{\theta}+\Omega_{e q}^{2} \sin \theta=0$

$\Omega_{e q}^{2}=M g \frac{\left(L_{1}-L_{2}\right)}{\left[3 m l^{2}+M\left(L_{1}^{2}+2 L_{2}^{2}\right)\right]}=\frac{g}{L_{2}} \frac{\gamma-1}{3 \psi+\gamma^{2}+2}$

$\psi=\frac{m l^{2}}{M L_{2}^{2}}, \quad \gamma=\frac{L_{1}}{L_{2}}>1$

If $M>>m$ the first term in the denominator $\psi$ can be neglected. Formula (3) indicates that the natural frequency of the system depends on the difference between $L_{1}$ and $L_{2}$ only. Figures 2 and 3 demonstrate the behaviour of the three equivalent natural frequencies $(\mathrm{Hz})$ of the system for $L_{2}=1$ $\mathrm{m}, L_{2}=3 \mathrm{~m}$ and $L_{2}=5 \mathrm{~m}$ correspondingly for the case of $M>>m$, so that $\psi=0$. It can be seen from Fig. 2 that some desired natural frequencies may be achieved in two different combinations for a given value of $L_{2}$, one of which provide very large value of the pendulum length $L_{1}$ (large values of $\gamma$ ). Moreover, the same frequency may be achieved for different values of $L_{1}$ and $L_{2}$, thus different torque can be created. It should also be mentioned that there is an upper boundary on the frequency which can be achieved by each pendulum size. Namely, the larger the distance $L_{2}$ the lower 


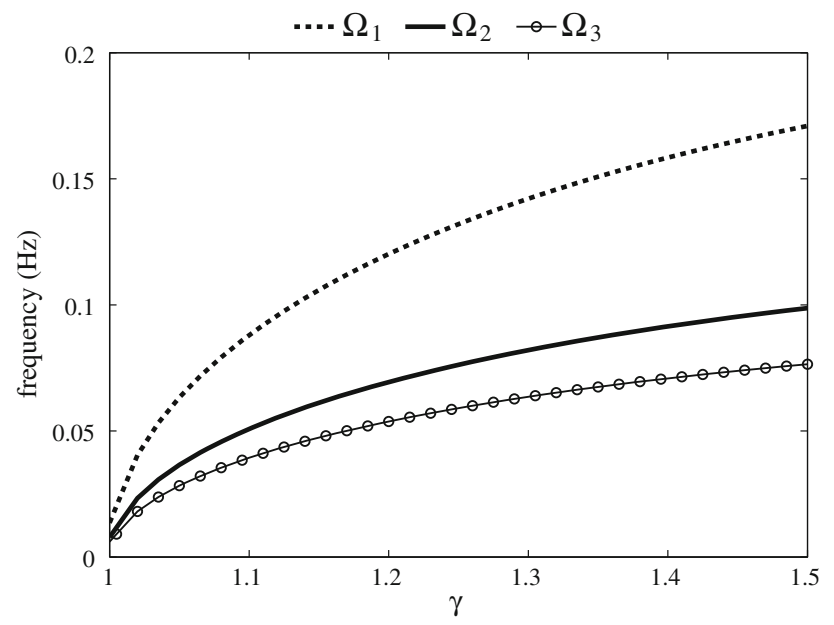

Fig. 3 Zoom in of Fig. 2

value of maximum frequency it can reach. Thus, the same frequency of $0.316 \mathrm{rad} / \mathrm{s}$ can be achieved.

At this point one can generalise the model to any odd number of equally spaced arms. The equation of motion of such a system may be written as:

$$
\begin{aligned}
& \quad\left[N m l^{2}+M\left(L_{1}^{2}+(N-1) L_{2}^{2}\right)\right] \ddot{\theta} \\
& =-M g L_{1} \sin \theta+M g L_{2} \sum_{i=1,2,3,}^{(N-1) / 2}\left\{\sin \left(\frac{2 i \pi}{N}-\theta\right)\right. \\
& \left.\quad-\sin \left(\frac{2 i \pi}{N}+\theta\right)\right\}, \\
& N=1,3,5,7, \ldots
\end{aligned}
$$

After simplification once again one arrives to the well-known pendulum Eq. (2) with the following value of the natural frequency:

$$
\begin{aligned}
\Omega_{e q F}^{2} & =M g \frac{L_{1}-L_{2}}{\left[N m l^{2}+M\left(L_{1}^{2}+(N-1) L_{2}^{2}\right)\right]} \\
& =\frac{g}{L_{2}} \frac{\gamma-1}{N \psi+\gamma^{2}+N-1}
\end{aligned}
$$

The even number of arms is much easier to analyse since the contribution to the restoring force will come only from two arms located opposite to each other (at $180^{\circ}$ ), one of length $L_{1}$ and the other $L_{2}$. Thus formula (5) for the equivalent natural frequency is valid for odd as well as even number of equally spaced arms. Figure 4 demonstrates the results for a different number of arms (two, three and five), the same value of $L_{2}=3 \mathrm{~m}$ and for the case of $M>>m$. As expected, the more arms the system has the less value of maximum frequency it may achieve.

It should be stressed that such a design is very easy to calibrate and it is not sensitive to small descrepancies of the

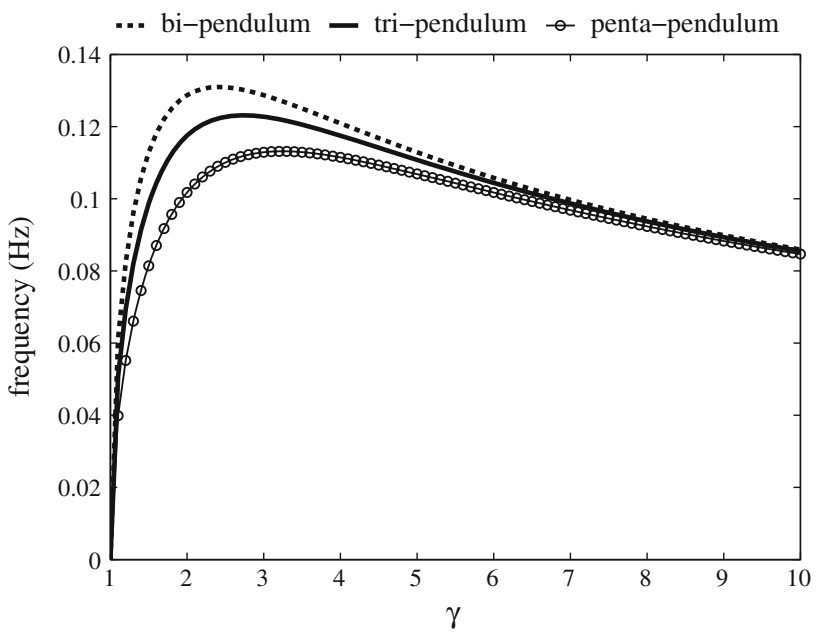

Fig. 4 Natural frequency of the pendulum with 2, 3 and 5 arms correspondingly for $L_{2}=3 \mathrm{~m}$

arms during the manufacturing process, since moving the lumped masses to a proper distance $\bar{L}_{1}$ would eliminate them.

\section{Application of the tri-pendulum for a wave energy power take-off system}

Wave energy converters (WECs) are used to convert wave energy to electricity. An important part of any WEC device is the power take-off (PTO) system, which actually provides a mechanism for extracting wave energy. Almost 40 years have passed since Salter [1] published his pioneering work on a wave energy PTO. Since then, various devices to capture wave energy have been developed. All of them may be classified in different ways and most of them may be found in the recent report by RenewableUK [2].

There are devices such as the heaving buoy, which benefit from using the vertical motion of waves. Some of them use a direct drive linear generator [3], others employ hydraulic rams or equivalent devices. So far there has been no wave energy converter which would generate electricity in a conventional way by spinning the rotor of an electric generator. In the paper by Xu et al [4] the authors outlined the possibility of using the parametric resonance phenomenon of a lumped mass pendulum having its pivot point vertically excited by waves, to create a wave PTO system. This idea was investigated numerically using the stochastic approach in [5] and it has been shown that in random sea environment it is still possible to achieve sustainable rotational motion of the pendulum. Although it is possible to generate electricity from an oscillatory motion, it leads to a number of problems related to converting a variable speed and variable voltage outcome. Rotational motion is also preferable since the kinetic energy stored in the system is bigger than that of oscillations. 
It is well-known that, if the pendulum's suspension point is oscillating in the vertical direction with a proper frequency, the pendulum will start rotating around its suspension point [4,6-13]. If the force driving the suspension point, is due to the heaving motion of waves, the device will be able to harvest wave energy and convert it into electricity. Similar idea of tuning to a specific wave frequency is used in a number of wave PTO. The system motion in this case is described by a nonlinear Mathieu equation [14]:

$\theta^{\prime \prime}+[1+\lambda \cos (\nu \tau)] \sin \theta=0$,

$\tau=\Omega t, \quad \Omega^{2}=\frac{g}{l}, \quad v=\frac{\omega}{\Omega}, \quad \lambda=\frac{A}{l} v^{2}$

where $\Omega$ natural frequency of the system, $l$ pendulum length, $A$ amplitude of waves, so that the wave height is twice the amplitude, $\omega$ wave frequency. For the primary parametric resonance one would need to regulate $\omega=2 \Omega$, so that $\lambda=$ $4 A / l$.

The system in Eq. (6) is a strongly nonlinear one. The subharmonic and homoclinic bifurcations of the parametric pendulum leading to unstable motion were studied in [8] using the Melnikov method. Bishop et al [6] showed the path from the symmetry-breaking of the stable oscillatory response to the rotational and Szemplinska-Stupnicka and Tyrkiel [7] conducted an extensive study on the oscillatory and rotatory attractors. The harmonic balance method and the critical velocity criterion were used in Clifford and Bishop [9] to approximate the escape zone of non-rotating orbits. In $\mathrm{Xu}$ and Wiercigroch [10] approximate analytical expressions of the resonance curves were presented utilizing the multiple scales method. Several studies $[4,11,12]$ reported the existence of rotational response of the parametric pendulum with approximate analytical solutions of the rotating orbits having been obtained in [13] using the pertrubation method. Moreover, Yurchenko et al [5] showed that rotational response is possible when the ocean waves are considered and modelled as a narrow-band stochastic process as well. A number of laboratory experiments have verified this idea, providing another possible wave energy conversion principle $[15,16]$

Unfortunately, this idea could not be implemented due to a significant drawback, related to the size of the system. Namely, the ocean waves have a frequency of about $f_{e}=0.1$ $\mathrm{Hz}$ or $\pi / 5 \mathrm{rad} / \mathrm{s}$ [17], which is several times lower than that used in the laboratory experiments. For the primary parametric resonance, the excitation frequency should be twice as much as the natural frequency of the system $f_{e}=2 f_{0}$. This leads to the following expression for the pendulum length:

$l=\frac{g}{\Omega^{2}}=\frac{g}{4 \pi^{2} f_{0}^{2}}=\frac{g}{\pi^{2} f_{e}^{2}} \approx 100 \mathrm{~m}$

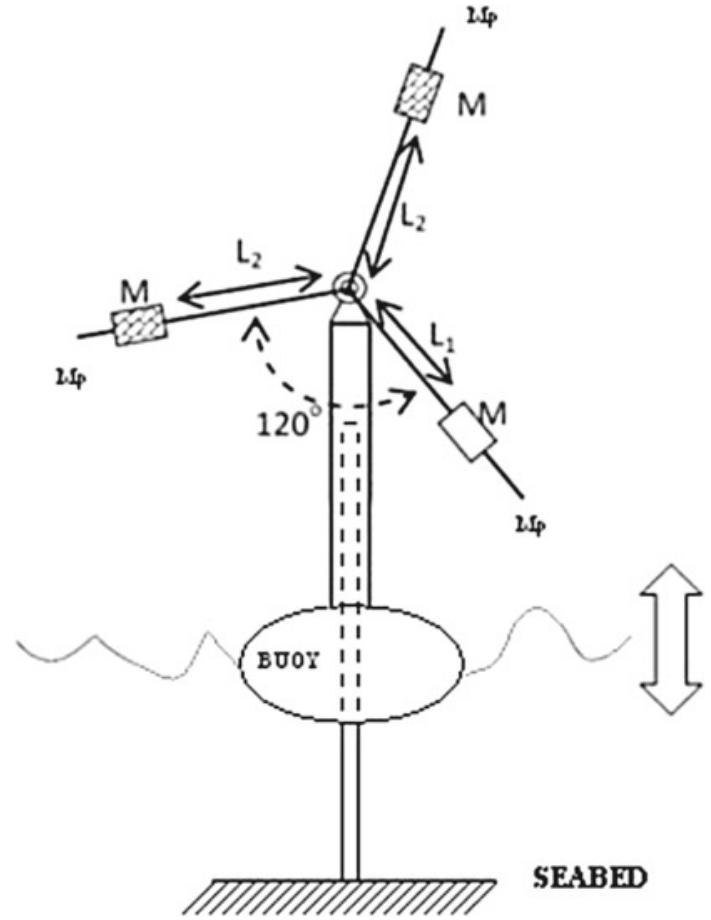

Fig. 5 Sketch of the proposed horizontal axis PTO

where $g$ acceleration due to gravity ${ }^{1}$. However, the numerical simulations, conducted by $\mathrm{Xu}$ et al. [4] for the deterministic system (6) revealed that the rotational motion of the pendulum is possible for values of $\lambda>1$.

The discussed shortcoming can be overcome using the design of the tri-pendulum, shown in Fig. 5. The main idea is that, from the dynamics point of view of an SDOF nonlinear system, the response of the parametric simple pendulum in the state space would be identical with the tri-pendulum's provided that $\Omega=\Omega_{e q}$, as it can be seen from (3). The major difference is that for a simple pendulum an unfeasible size would be required while a trivially-sized design of the tri-pendulum could match the requirements as demonstrated in Sect. 2.

Although in this work the authors consider strictly vertical motion of the buoy, in general the motion may be more complex, which incorporates inclinations from the vertical position (pitch angle) due to incoming and outgoing waves [18-20]. The tri-pendulum should be mounted onto a floating platform. For waves of slightly over $1 \mathrm{~m}$ in amplitude, one can select $L_{1}=2 \mathrm{~m}$ and $L_{2}=1.89 \mathrm{~m}$.

Furthermore, it should be stressed that the current design opens a wide range of possibilities, including capturing large waves and adjusting to a wave frequency by moving the lumped masses along the arms, using a threaded rod for instance.

$\overline{1}$ Usage of a physical pendulum is discussed in Sect. 1 
(a)

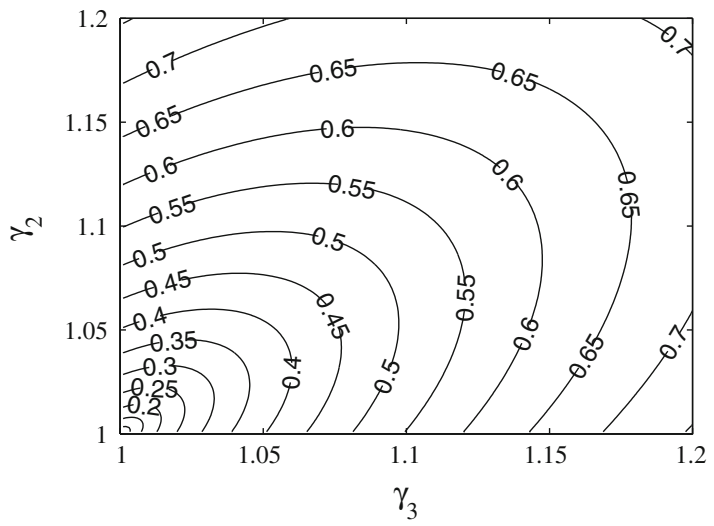

(c)

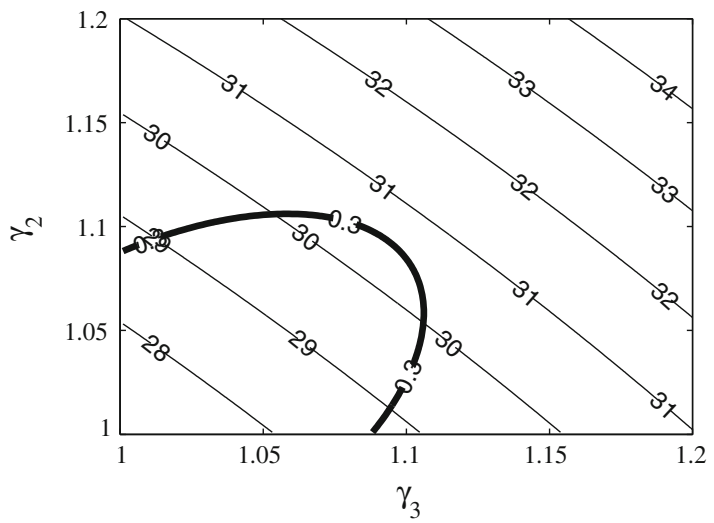

(b) $\mathrm{I} / \mathrm{M}$ for $\mathrm{L}_{1}=1$

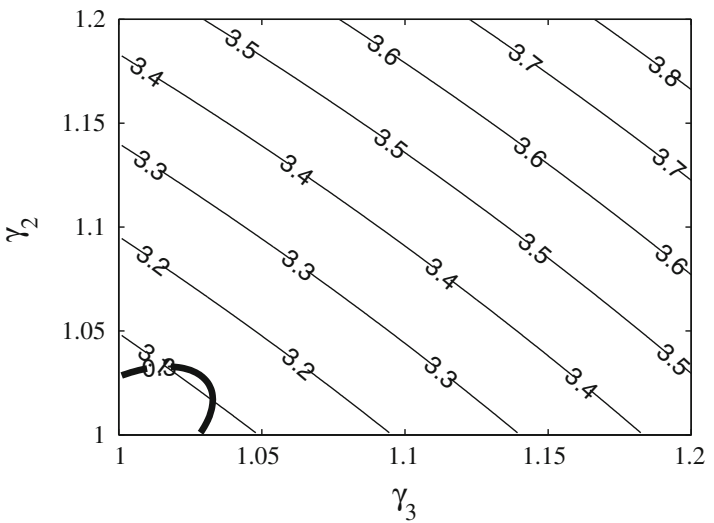

(d)

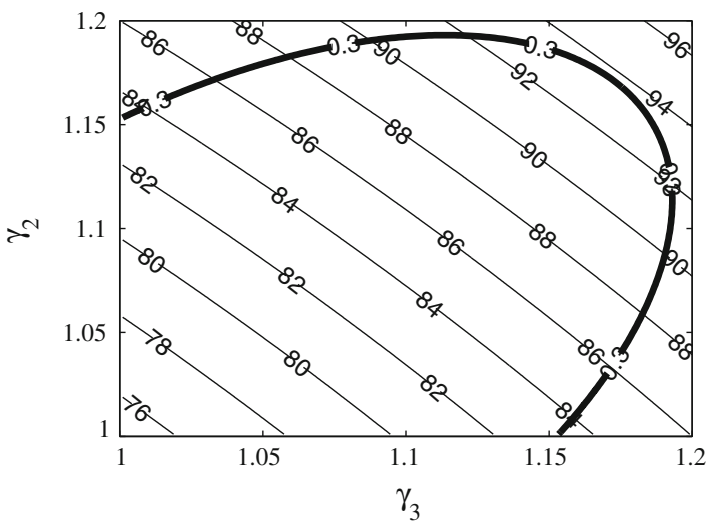

Fig. 6 a Natural frequency of the tripendulum with $L_{1} \neq L_{2} \neq L_{3}$ and $M_{i}=M$ with respect to the shortest length, $\Omega \sqrt{L_{1}}$, against $\gamma_{2}=L_{2} / L_{1}$ and $\gamma_{3}=L_{3} / L_{1}$; normalised inertia $I / M$ for $\mathbf{b} L_{1}=1 \mathrm{~m}, \mathbf{c} L_{1}=3 \mathrm{~m}$ and $\mathbf{d} L_{1}=5 \mathrm{~m}$

\section{Energy harvesting by different configurations of the tri-pendulum}

One might consider even more flexible configurations of the design regarding the arms' length and the carried masses. To that end, let us first consider the case of a tri-pendulum of equal lumped masses $M$ as introduced before, only that the arms' lengths are not constrained by $L_{2}=L_{3}$, leading to the more generic $L_{1} \neq L_{2} \neq L_{3}$. In the following, the length of the arms will be non-dimensionalised with respect to the length of the shortest arm. Since this is a parametric study, in each case presented one arm will be assumed as being the shortest and the others will be normalised with respect to it. This is a useful manipulation, because as it can be seen in Figs. 2 and 3 the resulting natural frequency is not the same for different sizes of the tri-pendulum, an indicator of which is the shortest arm's length. Then, correspondingly to the previous definitions, let $\gamma_{2}=L_{2} / L_{1}$ and $\gamma_{3}=L_{3} / L_{1}$. Following similar manipulations as for Eq. (3) and considering again the arms' masses negligible, one would end up with:

$$
\begin{aligned}
& \Omega_{e q}^{2}=\frac{g \sqrt{\left(1-\frac{\gamma_{2}+\gamma_{3}}{2}\right)^{2}+0.75\left(\gamma_{2}-\gamma_{3}\right)^{2}}}{L_{1}\left(1+\gamma_{2}^{2}+\gamma_{3}^{2}\right)} \\
& I=M L_{1}^{2}\left(1+\gamma_{2}^{2}+\gamma_{3}^{2}\right)
\end{aligned}
$$

where $I$ is the inertia of the rotating parts.

Figure 6a shows a contour plot of the equivalent natural frequency of the tri-pendulum with respect to what is considered as the shortest arm length, i.e. $\Omega \sqrt{L_{1}}$. It can be seen that the desired frequency range, around $0.314 \mathrm{rad} / \mathrm{s}$, could become easily achievable by regulating $L_{1}$ at values such as 2 or $3 \mathrm{~m}$, which are reasonably low from a structural point of view. However, another aspect for consideration would be the inertia of the rotating device and that is due to the fact that the bigger the inertia of the body in rotation the bigger the generator it could drive. Thus, whereas the manufacturing and maintenance processes favor an as small as possible size, energy production would benefit from larger sizes. In Fig. 6b-d, the normalised inertia of the rotating system over the mass $M$ of the lumped masses is presented for different values of 
(a)

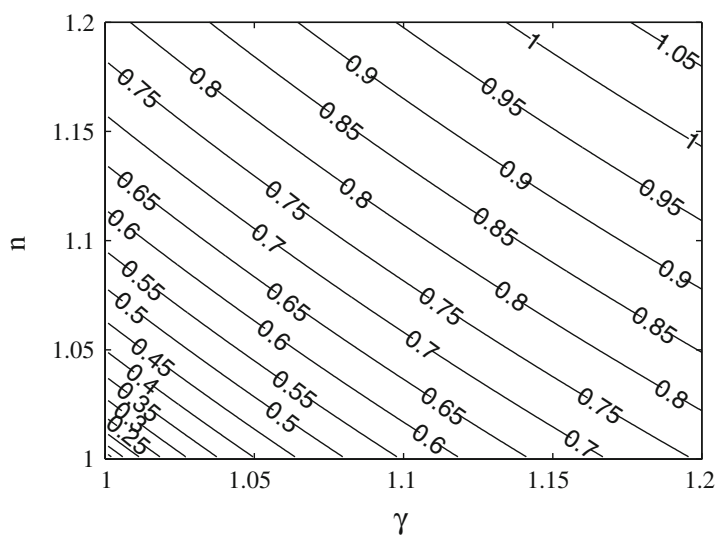

(c)

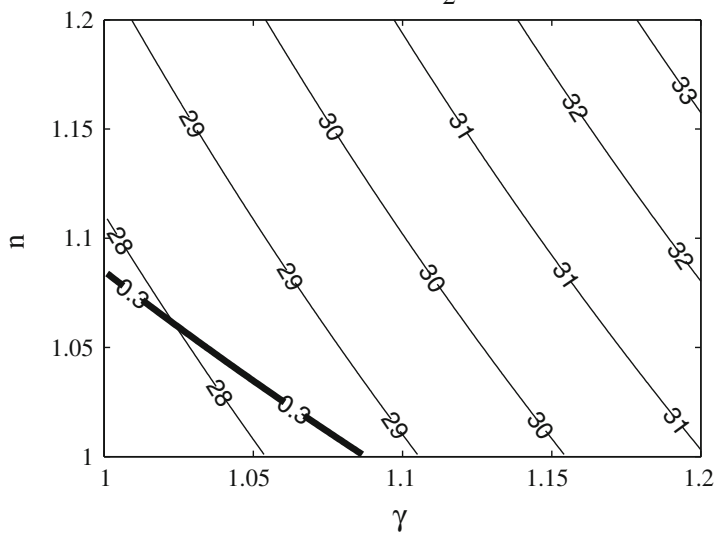

(b) $\mathrm{I} / \mathrm{M}$ for $\mathrm{L}_{2}=1$

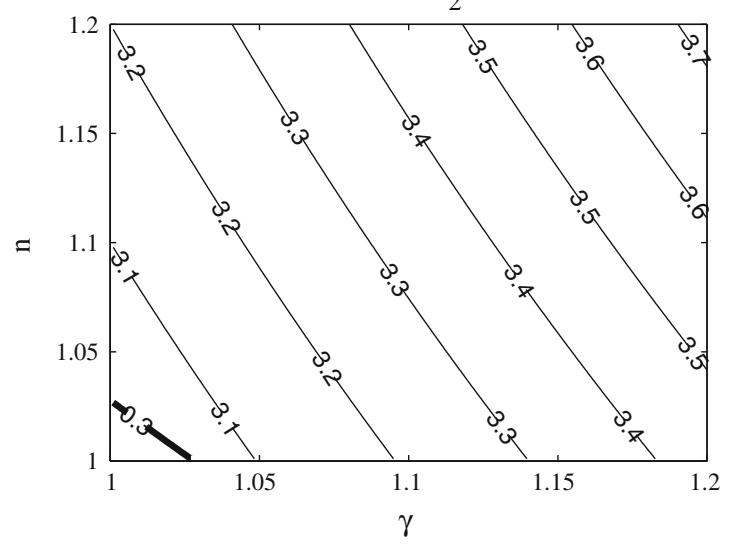

(d)

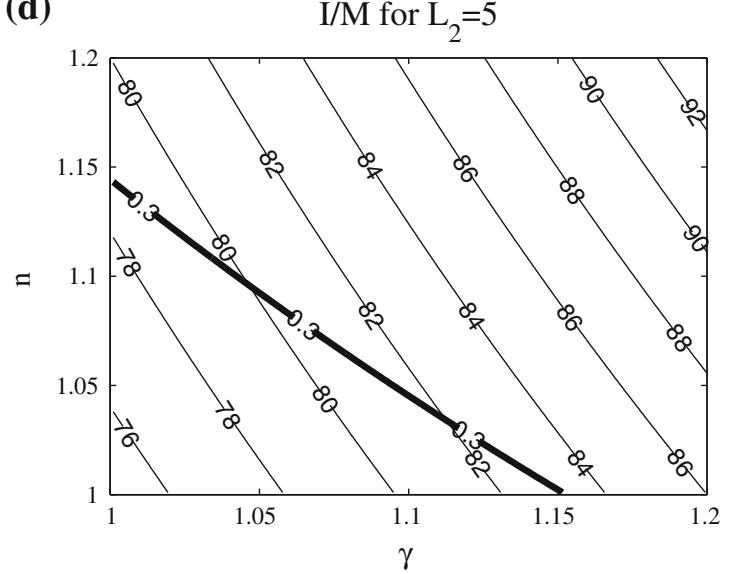

Fig. 7 a Natural frequency of the tripendulum with $L_{1}>L_{2}=L_{3}$ and $M_{1}>M_{2}=M_{3}$ with respect to the shortest length, $\Omega \sqrt{L_{2}}$, against $\gamma=L_{1} / L_{2}$ and $n=M_{1} / M_{2}$; normalised inertia, $I / M_{2}$ for: $\mathbf{b} L_{2}=1 \mathrm{~m}, \mathbf{c} L_{2}=3 \mathrm{~m}, \mathbf{d} L_{2}=5 \mathrm{~m}$

$L_{1}=1,3,5 \mathrm{~m}$. Along that, the target-frequency is plotted ( $\Omega=0.3 \mathrm{rad} / \mathrm{s}$ ) so as to attach the inertia values that could be achieved with such a design to the desired frequency area.

Obviously, the inertia of the rotor is proportional to the squared lengths of the arms so its value is increasing to the upper right of each figure. Let us now shortly discuss the impact of the inertia on the potentially produced energy. A main advantage of the proposed concept is the straightforward PTO system where a conventional generator is to be attached directly to the shaft of the rotor. This is important in a sense that no intermediate system is required for transforming the wave power to electrical other than a coupling and a gearbox in contrast with existing WEC technologies where it is rather common for hydraulic systems, linear generators or turbines to be used for ultimately producing electricity. Having the generator driven directly by the rotor's shaft would definitely decrease the complexity of the WEC. In that case, the generator would add in Eq. (2) a resisting torque which would be normalised by the rotor's inertia. So, the bigger the inertia that is excited by waves the bigger the generator that could be set to run by it.
Moving forward, let us now consider again a tri-pendulum where for the arms' lengths it holds $L_{1}>L_{2}=L_{3}$ as before, but now the corresponding relation holds for the lumped masses as well, i.e. $M_{1}>M_{2}=M_{3}$. Again, we define $\gamma=L_{1} / L_{2}$ and we introduce $n=M_{1} / M_{2}$. The natural frequency and the system's inertia in this case could be expressed by the following:

$$
\begin{aligned}
& \Omega_{e q}^{2}=\frac{g(n \gamma-1)}{L_{2}\left(n \gamma^{2}+2\right)} \\
& I=M_{2} L_{2}^{2}\left(n \gamma^{2}+2\right)
\end{aligned}
$$

Figure 7 presents the values for the normalised frequency and inertia against $\gamma$ and $n$ in the same manner as in Fig. 6. The natural frequency with respect to the shortest length which is now assumed to be $L_{2}, \Omega \sqrt{L_{2}}$, is plotted in Fig. 7a. The desired application-oriented frequency range is again achievable for reasonable values of $L_{2}$, fulfilling the structural requirements discussed previously. The rest of the plots, Fig. 7b-d, depict the variation of the normalised system iner- 
tia, $I / M_{2}$, along a short range of the parameters for different length values, $L_{2}=1,3,5 \mathrm{~m}$. The range of the inertia is seen to be similar with the previous case however the frequency follows rather linear isolines which slightly changes the possible matches of the targeted frequency with the system's inertia.

Closing this section, two remarks could be made upon the behaviour of the frequency with respect to the given parameters. First, from both Figs. 6a and 7a it is noticed that for bigger values of the shortest arm's length, the required ratios to achieve the same frequency increase as well. This means that the length increase is not proportional in between the arms and rather extreme values would be required should the shortest arm was to be heavily lengthened. Secondly, these extreme values could well enough introduce shocklike excitations of the pivot which would result in undesirable response of the rotor, let alone the thereafter structural concerns. In the same manner, it is fortunate that the sought frequency range is achievable for $n, \gamma, \gamma_{2}, \gamma_{3}$ ratio values close to 1 and thus extreme phenomena are avoided.

Performing a rough estimation of the mechanical power harvested from ocean waves by use of the tri-pendulum, consider the design as described last with $n=M_{1} / M_{2}$ and $\gamma=L_{1} / L_{2}$ and suppose $M_{2}=500 \mathrm{~kg}$ and $L_{2}=5 \mathrm{~m}$. That would direct us to Fig. $7 \mathrm{~d}$. In order to approach the necessary natural frequency one would have to choose $n, \gamma \approx 1.1$ leading to $I / M_{2} \approx 83 \mathrm{~m}^{-2}$. Taking into account that the mechanical power of the rotor could be expressed as $P=I \ddot{\theta} \dot{\theta}$ and considering a period-1 rotational point of the parameters $(\nu, \lambda)$, then the average power extracted by the heaving motion of waves and transformed to mechanical power of the rotor would be around $4 \mathrm{~kW}$.

Scaling up the device for practical applications shows that it is possible to achieve much higher inertia values (see Fig. 8) therefore leading to greater power generation, however in this case one mass should be several times larger than the others and should be positioned far away from them, an extreme case of $n=10, \gamma=10$ shown in Fig. 8 being an example. In this configuration the desired natural frequency would be achieved only by applying unrealistic sizes due to the constrain imposed by the frequency of the waves. This fact clearly states that in the scaling process the tri-pendulum tends to a structure of a regular pendulum.

\section{Discussion of an experimental rig}

Parametric excitation of a pendulum has attracted significant interest of the scientific community regarding the stability boundaries, chaotic attractors and the bifurcations that have been observed. It is only reasonable that experimental investigation has been part of the ongoing research with the scope varying from identification of the

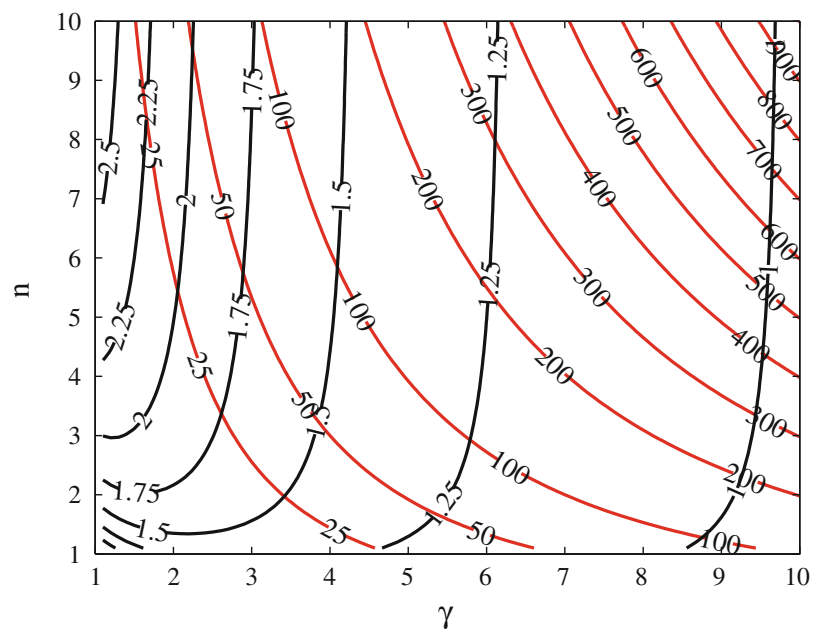

Fig. 8 Parametrised natural frequency, $\Omega \sqrt{L_{2}}$, (black solid line) and inertia, $I /\left(M_{2} L_{2}^{2}\right),($ red solid line $)$ against large range of $\gamma=L_{1} / L_{2}$ and $n=M_{1} / M_{2}$. (Color figure online)

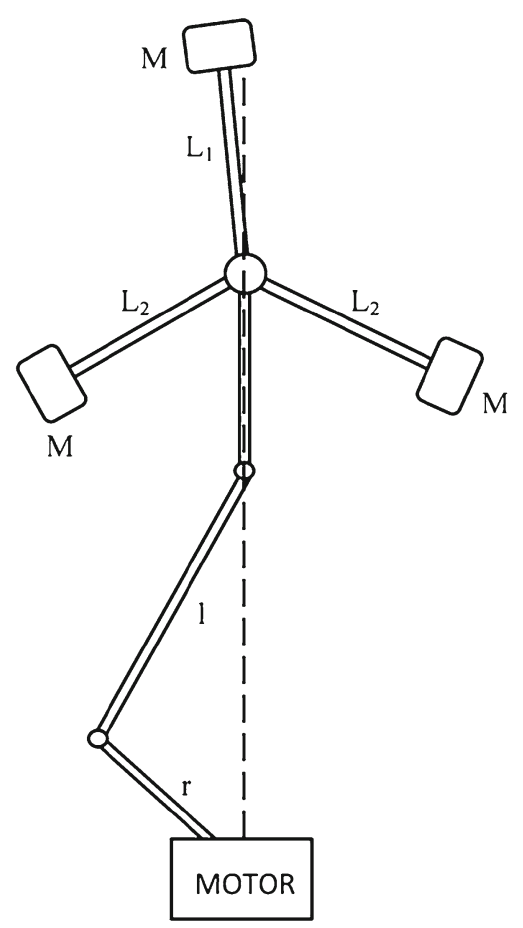

Fig. 9 Sketch of the experimental rig

chaotic attractors $[21,22]$ to the bifurcations occurring in an inverted pendulum [23] or even a double pendulum [24]. In the frame of developing a pendulum-based WEC, rotational response of the parametric pendulum driven by a shaker was sought [15] and a benchmark-proof of concept-experimental study was conducted in a wave tank [16].

However, most of the previous experiments concentrated on considerably higher frequencies than those observed in ocean waves. At the time, an experimental rig of an ongoing 

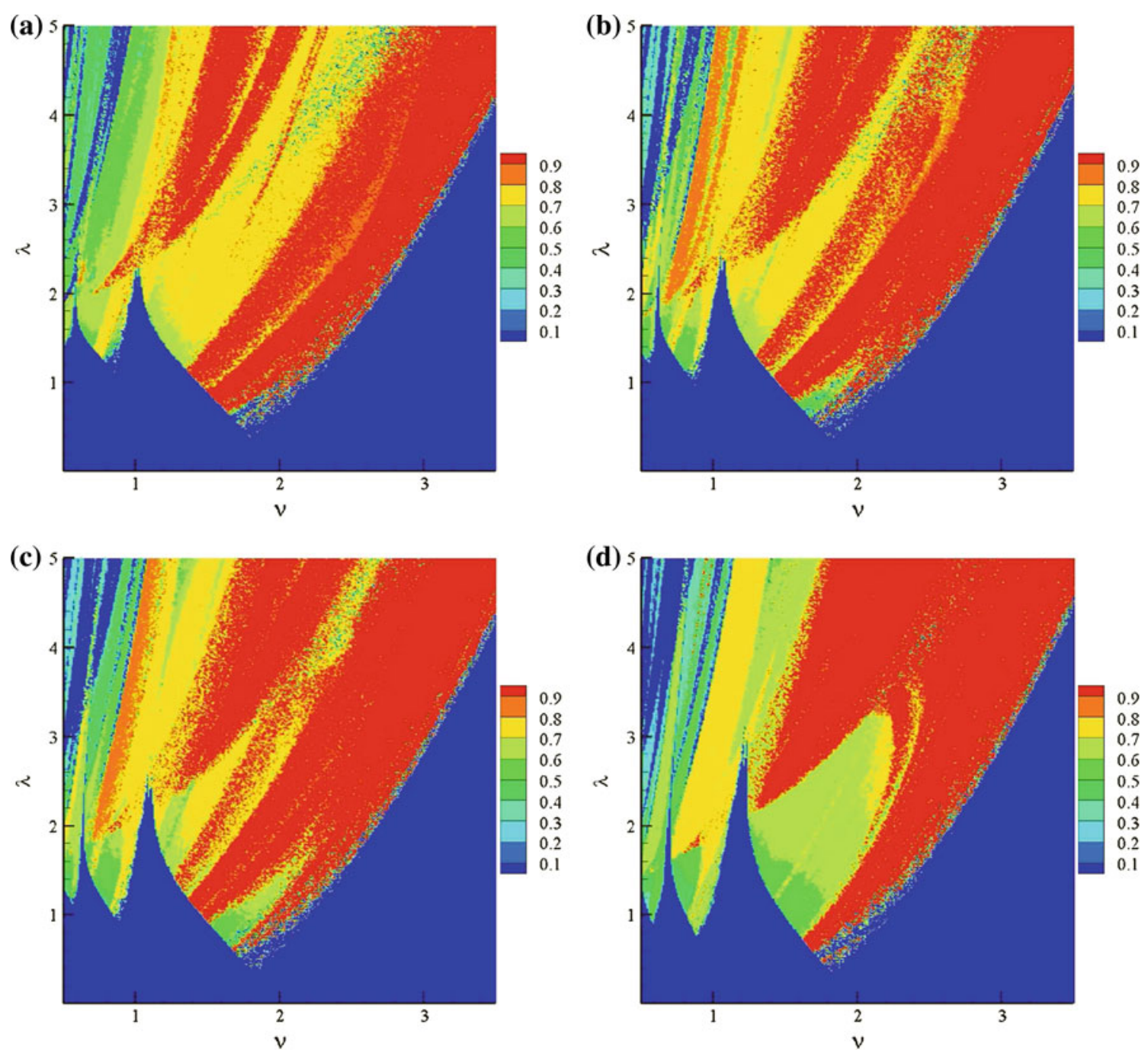

Fig. 10 Parameter space plots for a physical pendulum parametrically excited by Eq. (10) for $\mathbf{a} j=2, \mathbf{b} j=3$, c $j=4$ and $\mathbf{d} j=\infty$

investigation is under development aiming to demonstrate the functionality of a tri-pendulum as it was introduced in the previous sections. The exciting force is designed to simulate ocean waves by applying frequencies and amplitudes much closer to those occurring in nature.

Figure 9 shows a sketch of the rig. Namely, the tripendulum will be attached to a vertical rod allowed to move in one direction constrained by a slider-rail system functioning as a linear bearing. The total achievable stroke will be over $1 \mathrm{~m}$ in order to approach amplitudes close to the ones exhibited in sea. At the bottom of that rod, a connecting rod of a reciprocal piston-like mechanism will be hinged. Its other endpoint will perform a circular motion driven by a crank of adjustable length and rigidly connected to the shaft of a motor. It is well known that the working point of that mechanism which could be also thought as the translation of the pendulum's pivot will follow a sinusoidal profile augmented by subharmonic excitations depending on the ratio of the length of the connecting rod over the crank's one. The acceleration of the pivot in this case can be fairly approximated by the following expression:

$\ddot{x}=\omega^{2} r\left(\cos \omega t+\frac{\cos 2 \omega t}{j}\right)$

where $\omega$ the motor's speed in $\mathrm{rad} / \mathrm{s}, r$ the crank's radius, $j=l / r$ the size ratio and $l$ the length of the connecting rod. Obviously, in the limit case of $j \rightarrow \infty$ the excitation reduces to a perfect sinusoidal as in Eq. (6). It is also seen that the speed of the motor defines the excitation frequency and the crank radius the amplitude. In this paper, the response of a tri-pendulum carrying equal masses $M$ and excited by Eq. (10) is modelled by means of numerical analysis and parameter space plots are constructed describing the amount of the exhibited rotational motion. Then, the governing equation would be: 
$\theta^{\prime \prime}+c \theta^{\prime}+\left[1+\lambda\left(\cos v \tau+\frac{\cos 2 \nu \tau}{j}\right)\right] \sin \theta=0$

where $c$ denotes the coefficient of viscous damping. Figure 10 shows the parameter space plots calculated for different values of the size ratio. In these figures, red colors the parameter regions where over $90 \%$ of rotations were observed while blue those with less than $10 \%$. Here, the period-doubling bifurcations are not considered and thus period-1, period-2 and so on responses are not separately displayed nor do different attractors such as the chaotic ones. The target of this investigation is to identify the parameters that would lead to rotational motion of the tri-pendulum simulating the vertical translation imposed by the described rig's reciprocal mechanism. The damping coefficient is kept constant for all the plots taking a small value, $c=0.01$, approaching the one of the experimental setup's.

Figure 10d assumes a size ratio $j \rightarrow \infty$ practically devolving to the perfect harmonic excitation described in Eq. (6). This plot resembles as expected the ones previously created for the perfect harmonic signal [5] and is shown to facilitate comparison. Further on, three values for the size ratio $j=2,3,4$ are considered and the corresponding plots are shown in Fig. 10a-c. A first observation would be that the boundaries separating oscillatory and asymptotically stable motion from the rotary and chaotic ones remain almost independent of the parameter $j$ something that especially holds for the primary resonance zone around $v=2$. However, the picture is rather different when it comes to rotational and chaotic regions. While in the purely harmonically excited case the two rotational regions merge for $\lambda>3.2$, they remain completely distinct for $j=2$ shown in Fig. 10a, separated by a long chaotic attractor. Increasing the size ratio reshapes the plots towards the limiting case of $j \rightarrow \infty$ retaining though distinguishable differences in the internal structure of the map. Thus, an experimental investigation as the one described that would be based on Eq. (6) and not take into account the subharmonic excitation, would reflect significantly erroneous results depending on the selected size ratio $j$.

\section{Conclusions}

This paper offers a design of the N-pendulum, which can be considered as a special case of a physical pendulum. Nevertheless, the proposed $\mathrm{N}$-pendulum is a much better choice for some applications and in particular for the wave energy harvesting application, because it is capable of not only achieving low frequencies staying relatively small in size, but also being able to easily control its natural frequency and the created torque. It has been shown that the proposed design can be used as the first horizontal axis wave PTO system. Besides, other major advantages of the proposed design are its simplicity, possible automatic calibration and simple start-stop mechanism, all of which are important features of energy converters. Although the numerical results for the system's response have been provided, their experimental validation is required. For this purpose the proposed design has been assembled and about to be tested at the Heriot-Watt University.

\section{References}

1. Salter S (1974) Wave power. Nature 249:720-724

2. Matthews S, Adams J, Valpy B, Krohn D (2012) Marine energy in the UK. State of the industry report 2012. Technical Report, RenewableUK

3. Ran L, Mueller M, Ng C, Tavner P, Zhao H, Baker N, Mcdonald S, Mckeever P (2007) Power conversion and control for a linear direct drive permanent magnet generator for wave energy. IET Renew Power Gener 5:1-9

4. Xu X, Wiercigroch M, Cartmell M (2005) Rotating orbits of a parametrically-excited pendulum. Chaos Solitons Fractals 23:1537-1548

5. Yurchenko D, Naess A, Alevras P (2013) Pendulum's rotational motion governed by a stochastic Mathieu equation. Probab Eng Mech 31:12-18

6. Bishop SR, Sofroniou A, Shi P (2005) Symmetry-breaking in the response of the parametrically excited pendulum. Chaos Solitons Fractals 25:257-264

7. Szemplinska-Stupnicka W, Tyrkiel E (2002) The oscillationrotation attractors in the forced pendulum and their peculiar properties. Int J Bifurcation Chaos 12(01):159-168

8. Koch BP, Leven RW (1985) Subharmonic and homoclinic bifurcations in a parametrically forced pendulum. Physica 16D:1-13

9. Clifford MJ, Bishop SR (1994) Approximating the escape zone for the parametrically excited pendulum. J Sound Vib 172(4):572-576

10. Xu X, Wiercigroch M (2007) Approximate analytical solutions for oscillatory and rotational motion of a parametric pendulum. Nonlinear Dyn 47:311-320

11. Clifford MJ, Bishop SR (1995) Rotating periodic orbits of the parametrically excited pendulum. Phys Lett 201A:191-196

12. Garira W, Bishop SR (2003) Rotating solutions of the parametrically excited pendulum. J Sound Vib 263:233-239

13. Lenci S, Pavlovskaia E, Rega G, Wiercigroch M (2008) Rotating solutions and stability of parametric pendulum by perturbation method. J Sound Vib 310:243-259

14. Nayfeh A, Mook D (1979) Nonlinear oscillations. Wiley, New York

15. Xu X, Pavloskaia E, Wiercigroch M, Romeo F, Lenci S (2007) Dynamic interactions between parametric pendulum and electrodynamical shaker. ZAMM 87:172-186

16. Lenci S, Brocchini M, Lorenzoni C (2012) Experimental rotations of a pendulum on water waves. J Comput Nonlinear Dyn 7(1):011007

17. Cruz J (2008) Ocean wave energy. Current status and future perspectives. Springer, Berlin

18. Horton B, Sieber J, Thompson J, Wiercigroch M (2011) Dynamics of the nearly parametric pendulum. Int J Nonlinear Mech 46:436442

19. Belyakov A (2011) On rotational solutions for elliptically excited pendulum. Phys Lett A 375:2524-2530

20. Pavlovskaia E, Horton B, Wiercigroch M, Lenci S, Rega G (2011) Approximate rotational solutions of pendulum under com- 
bined vertical and horizontal excitation. Int $\mathrm{J}$ Bifurcation Chaos 22(5): 1250100

21. Leven R, Koch B (1981) Chaotic behaviour of a parametrically exited damped pendulum. Phys Lett A 86:71-74

22. Leven R, Pompe B, Wilke C, Koch B (1985) Experiments on periodic and chaotic motions of a parametrically forced pendulum. Phys D Nonlinear Phenom 16(3):371-384
23. Yabuno H, Miura M, Aoshima N (2004) Bifurcation in an inverted pendulum with tilted high-frequency excitation: analytical and experimental investigations on the symmetry-breaking of the bifurcation. J Sound Vib 273:493-513

24. Sartorelli C, Lacarbonara W (2012) Parametric resonances in a base-excited double pendulum. Nonlinear Dyn 69:1679-1692 